\title{
DECISION-MAKING OF PORTFOLIO INVESTMENT WITH LINEAR PLUS DOUBLE EXPONENTIAL UTILITY FUNCTION $^{*}, * *$
}

\author{
Qinguian Zhou ${ }^{1,2}$, Jia JiaO ${ }^{2}$, Datian Niu ${ }^{2}$ And Deli Yang $^{1}$
}

\begin{abstract}
This paper broadens the exponential utility function commonly used by risk-averse investors to the linear plus double exponential utility function, which is applicable in most cases. Thus it is of essential and supreme significance to conduct a research on its optimal investment portfolio in securities investment. This paper, by means of the non-difference curve method, carries out a research into the optimal portfolio decision-making by investors who have this type of utility function. The optimal decision-making and the ratio of optimal portfolio investment are derived. Finally, an actual case is given to verify the relevant results.
\end{abstract}

Keywords. Linear plus double exponential utility function, optimal portfolio, investment decision-making, non-difference curve method.

Mathematics Subject Classification. 91B28, 90B50.

Received April 27, 2012. Accepted July 11, 2013.

* This work is supported by NSFC (11001039,11226157), Independent Research Foundation of the Central Universities (DC110103, DC110325,DC13010319), Dalian Nationalities University DSF 20096209.

** Corresponding author: Qingjian Zhou, Jia Jiao.

1 Institute of Systems Engineering, Dalian University of Technology, Dalian 116024, P.R. China. zhouqingjian68@163.com; somdyang@dlut.edu.cn

2 College of Science, Dalian Nationalities University, Dalian 116600, P.R. China.

jiaolinrui@yahoo.cn; niudt@dlnu.edu.cn 


\section{INTRODUCTION}

In the portfolio investment of securities, each investor has his own utility function to express his satisfaction of the investment returns and tries to choose the optimal portfolio according to the principle of expected utility maximization. At the same time, most investors are rational risk-averse investors, that is to say, they will try to avoid relevant risk while seeking their returns. This paper broadens the exponential utility function widely used by risk-averse investors to the linear plus double exponential utility function, which expresses itself with $U=k_{1}+k_{2} x-\mathrm{e}^{-k_{3} x}-\mathrm{e}^{-k_{4} x}$. Here $x$ denotes investment return, and $k_{i}>0, i=1,2,3,4$. The utility functions of negative exponential type, linear plus exponential type and double exponential type can all be regarded as its special cases $[1,7-9]$, so it is of great importance to conduct a research on its optimal investment portfolio in securities investment.

First of all, let us examine the type of linear plus exponential utility function. Based on $k_{i}>0, \quad i=1,2,3,4$, we can conclude that $U^{\prime}=k_{2}+k_{3} \mathrm{e}^{-k_{3} x}+$ $k_{4} \mathrm{e}^{-k_{4} x}>0$ and $U^{\prime \prime}=-k_{3}{ }^{2} \mathrm{e}^{-k_{3} x}-k_{4}{ }^{2} \mathrm{e}^{-k_{4} x}<0$. For this reason, it is a riskaverse utility function. In most cases, when investors are about to choose a certain portfolio investment, they may take advantage of the linear plus exponential utility function in order to maximize the long-term return rate by maximizing the expected utility of each period.

In this paper the non-difference curve method (from now on NDCM) is applied to carry out a research into the optimal portfolio decision-making of investment. It is organized as follows: in Section 2 we provide an introduction to the Mean-Variance Model suggested by Markowitz. In Section 3, we apply NDCM to explore the decision-making in optimal portfolio of the linear plus exponential utility function and obtain the optimal decision-making. The actual practice of the research conclusion is presented in Section 4. Conclusions and final remarks follow in Section 5.

\section{The Mean-Variance model of markowitz}

Early in the 1950s, the American Economist and Financier H. Markowitz proposed his Portfolio Investment Theory, which was regarded as the theoretical foundation when solving such problems. His theory suggests that investors will determine the optimal portfolio with the purpose of enhancing their returns and reducing the risk. It is on this particular basis that Markowitz established his well-known Mean-Variance Model [2].

Suppose there are $n$ kinds of securities, whose prices at the current moment $(t=0)$ are $S_{10}, S_{20}, \ldots, S_{n 0}$, and at a future moment $S_{1}, S_{2}, \ldots, S_{n}$. Denote

$$
x_{i}=\ln \left(\frac{S_{i}}{S_{i 0}}\right), \quad i=1,2, \ldots, n, \quad X_{n \times 1}=\left(x_{1}, x_{2}, \ldots, x_{n}\right)^{T},
$$


where $x_{i}$ is the logarithm return rate of the $i$ th security during the period from the time $t=0$ to $t=T, X$ is the vector of logarithm return rate of all these $n$ kinds of securities. In the two formulas, $S_{10}, S_{20}, \ldots, S_{n 0}$ are known constants, and $S_{1}, S_{2}, \ldots, S_{n}$ are random variables, so $x_{1}, x_{2}, \ldots, x_{n}$ are random vectors and $X$ is a random vector. The return expectation and covariance matrix are represented respectively by the two formulas

$$
E X=\left(E x_{1}, E x_{2}, \ldots, E x_{n}\right)^{T}=\mu, \quad \operatorname{Var}(X)=E(X-E X)(X-E X)^{T}=\Sigma .
$$

Let $\omega_{i}(i=1,2, \ldots, n)$ denote the investment proportion of the $i$ th security, $\omega_{n \times 1}=$ $\left(\omega_{1}, \omega_{2}, \ldots, \omega_{n}\right)^{T}, e_{n \times 1}=(1,1, \ldots, 1)^{T}$ and the investment proportion must fulfill the condition $\omega^{T} e=1$. The investor's return $R=\omega^{T} X$ is one random variable. The expectation return and variance of $R$ are $r=E R=\omega^{T} \mu$ and $\sigma^{2}=\operatorname{Var}(R)=$ $\omega^{T} \Sigma \omega$, respectively.

The major concern of Markowitz's Mean-Variance Model is the problem of constrained extreme value:

(1) Under the condition of $\omega^{T} \mu=r_{0}$, figure out $\omega$ to minimize the risk, i.e. to minimize $\operatorname{Var}\left(\omega^{T} X\right)=\omega^{T} \Sigma \omega$.

(2) Under the condition of $\omega^{T} \Sigma \omega=\sigma_{0}^{2}$, figure out $\omega$ to maximize the return, i.e. to maximize $\omega^{T} \mu$.

Obviously the two problems are equivalent, and we choose to examine the former. So it boils down to a mathematic problem: to figure out the minimum of $\omega^{T} \Sigma \omega$ under the condition $\omega^{T} e=1$ and $\omega^{T} \mu=r_{0}$.

And then we can get the optimal portfolio investment proportion:

$$
\omega_{*}=\frac{C-r_{0} B}{\Delta} \Sigma^{-1} e+\frac{r_{0} A-B}{\Delta} \Sigma^{-1} \mu
$$

and the corresponding variance is

$$
\sigma^{2}\left(\omega_{*}\right)=\omega_{*}^{T} \Sigma \omega_{*}=\frac{1}{\Delta}\left(A r_{0}^{2}-2 B r_{0}+C\right),
$$

which we define it as the efficient frontier of the investment portfolio, where

$$
A=\mathrm{e}^{T} \Sigma^{-1} e, \quad B=\mathrm{e}^{T} \Sigma^{-1} \mu=\mu^{T} \Sigma^{-1} e, \quad C=\mu^{T} \Sigma^{-1} \mu, \quad \Delta=A C-B^{2} .
$$

\section{The STUdy of PORTFOLIO INVESTMENT WiTH LINEAR PLUS DOUBLE EXPONENTIAL UTILITY FUNCTION}

\subsection{Solution to its EU}

Here we take the hypothesis that investment returns follow a Guass distribution $N\left(r, \sigma^{2}\right)$, and $r, \sigma^{2}$ are defined as before. The density function of Guass distribution is

$$
f(x)=\frac{1}{\sqrt{2 \pi} \sigma} \mathrm{e}^{-\frac{(x-r)}{2 \sigma^{2}}}
$$


and hence the expectation of the linear plus double exponential utility function is

$$
\begin{aligned}
E U= & \frac{1}{\sqrt{2 \pi} \sigma} \int_{-\infty}^{\infty}\left(k_{1}+k_{2} x-\mathrm{e}^{-k_{3} x}-\mathrm{e}^{-k_{4} x}\right) \mathrm{e}^{-\frac{(x-r)^{2}}{2 \sigma^{2}}} \mathrm{~d} x \\
= & \frac{1}{\sqrt{2 \pi} \sigma} \int_{-\infty}^{\infty}\left(k_{1}+k_{2}(\sigma y+r)-\mathrm{e}^{-k_{3}(\sigma y+r)}-\mathrm{e}^{-k_{4}(\sigma y+r)}\right) \mathrm{e}^{-\frac{y^{2}}{2}} d(\sigma y+r) \\
= & {\left[\frac{1}{\sqrt{2 \pi}} \int_{-\infty}^{\infty}\left(k_{1}+k_{2} r\right) \mathrm{e}^{-\frac{y^{2}}{2}} \mathrm{~d} y+k_{2} \sigma \int_{-\infty}^{\infty} y \mathrm{e}^{-\frac{y^{2}}{2}} \mathrm{~d} y-\int_{-\infty}^{\infty} \mathrm{e}^{-k_{3}(y \sigma+r)-\frac{y^{2}}{2}} \mathrm{~d} y\right.} \\
& \left.-\int_{-\infty}^{\infty} \mathrm{e}^{-k_{4}(y \sigma+r)-\frac{y^{2}}{2}} \mathrm{~d} y\right] \\
= & \frac{1}{\sqrt{2 \pi}}\left[\sqrt{2 \pi}\left(k_{1}+k_{2} r\right)-\sqrt{2 \pi} \exp \left(-k_{3} r+\frac{k_{3}^{2} \sigma^{2}}{2}\right)-\sqrt{2 \pi} \exp \left(-k_{4} r+\frac{k_{4}^{2} \sigma^{2}}{2}\right)\right] \\
= & k_{1}+k_{2} r-\exp \left(-k_{3} r+\frac{k_{3}^{2} \sigma^{2}}{2}\right)-\exp \left(-k_{4} r+\frac{k_{4}^{2} \sigma^{2}}{2}\right) .
\end{aligned}
$$

\subsection{NON-DIFFERENCE CURVE}

As this type of utility function is averse to risk, we may employ NDCM in the field of investments for a possible solution. According to NDCM theory [3-6], nondifference curves (actually a set of equivalent utility curves) indicate the investor's preference when faced with the two alternatives. So we adopt the $r-\sigma^{2}$ coordinate system, in which the horizontal axis represents risk with the deviation $\sigma^{2}$ to measure it, and the vertical axis represents return with the expected return $r$ to measure it. Each investor has his own non-difference curve to indicate his preference for expected return and deviation. The investor's non-satisfaction and riskaverseness lead to this effect: the risk-averse non-difference curve tilts upper right as a downward convex. Besides, the utility of the non-difference curve family is increasing from bottom to top. So the $E U$ of linear plus double exponential utility function are one family of non-difference curves.

\subsection{The COnstruction of optimal DeCision-MAKing MODEL}

According to the principle of the Mean-Variance Theory, the optimal portfolio is the cut-off point of one non-difference curve with the efficient frontier curve. We may construct the following model:

$$
\left\{\begin{array}{l}
E U=k_{1}+k_{2} r-\exp \left(-k_{3} r+\frac{k_{3}^{2} \sigma^{2}}{2}\right)-\exp \left(-k_{4} r+\frac{k_{4}^{2} \sigma^{2}}{2}\right), \\
\sigma^{2}=\frac{1}{\Delta}\left(A r^{2}-2 B r+C\right),
\end{array}\right.
$$

as shown in Figure 1. 


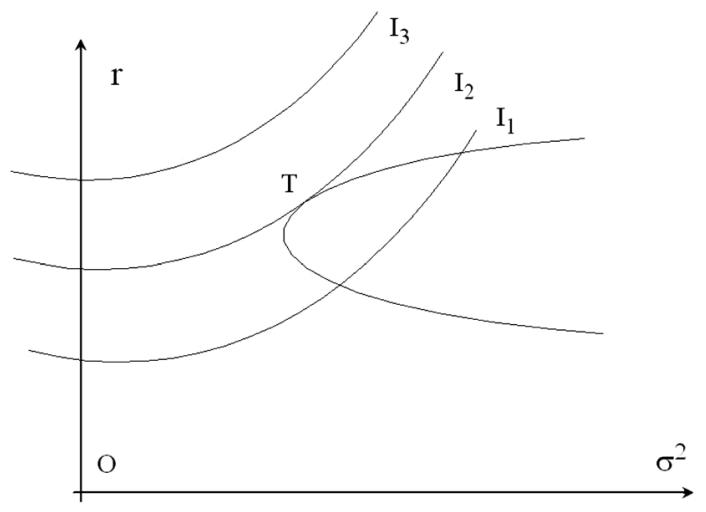

FiguRE 1. Optimal portfolio selection.

\subsection{The SOlution of OPtimal DECISION-MAKING}

Now we conduct a specific analysis of the above mentioned model. If we put efficient frontier $\sigma^{2}$ into expectation utility $E U$, then we will get

$$
\begin{aligned}
E U= & k_{1}+k_{2} r-\exp \left(-k_{3} r+\frac{k_{3}^{2}\left(A r^{2}-2 B r+C\right)}{2 \Delta}\right) \\
& -\exp \left(-k_{4} r+\frac{k_{4}^{2}\left(A r^{2}-2 B r+C\right)}{2 \Delta}\right) .
\end{aligned}
$$

We must figure out the maximum of the expected utility and its optimal proportion. As $k_{i}>0(i=1,2,3,4)$,

$$
\begin{gathered}
\frac{d E U}{d r}=k_{2}-\exp \left(-k_{3} r+\frac{k_{3}^{2}\left(A r^{2}-2 B r+C\right)}{2 \Delta}\right)\left(-k_{3}+\frac{k_{3}^{2}(A r-B)}{\Delta}\right) \\
-\exp \left(-k_{4} r+\frac{k_{4}^{2}\left(A r^{2}-2 B r+C\right)}{2 \Delta}\right)\left(-k_{4}+\frac{k_{4}^{2}(A r-B)}{\Delta}\right) \\
\frac{d^{2} E U}{d r^{2}}=-\exp \left(-k_{3} r+\frac{k_{3}^{2}\left(A r^{2}-2 B r+C\right)}{2 \Delta}\right)\left(-k_{3}+\frac{k_{3}^{2}(A r-B)}{\Delta}\right)^{2}+\left(\frac{k_{3}^{2} A}{\Delta}\right) \\
-\exp \left(-k_{4} r+\frac{k_{4}^{2}\left(A r^{2}-2 B r+C\right)}{2 \Delta}\right)\left(-k_{4}+\frac{k_{4}^{2}(A r-B)}{\Delta}\right)^{2}+\left(\frac{k_{4}^{2} A}{\Delta}\right)<0,
\end{gathered}
$$

the expected utility $E U$ has its maximum.

Let $\frac{d E U}{d r}=0$. Arrange it and get

$$
\begin{aligned}
k_{2}= & \exp \left(-k_{3} r+\frac{k_{3}^{2}\left(A r^{2}-2 B r+C\right)}{2 \Delta}\right)\left(-k_{3}+\frac{k_{3}^{2}(A r-B)}{\Delta}\right) \\
& +\exp \left(-k_{4} r+\frac{k_{4}^{2}\left(A r^{2}-2 B r+C\right)}{2 \Delta}\right)\left(-k_{4}+\frac{k_{4}^{2}(A r-B)}{\Delta}\right) .
\end{aligned}
$$


If we suppose the right side of the equation to be $z(r)$, then we can conclude that $z$ can be zero, i.e. the equation $z=0$ has its root, then

$$
\begin{aligned}
& \exp \left(-k_{3} r+\frac{k_{3}^{2}\left(A r^{2}-2 B r+C\right)}{2 \Delta}\right)\left(-k_{3}+\frac{k_{3}^{2}(A r-B)}{\Delta}\right) \\
& +\exp \left(-k_{4} r+\frac{k_{4}^{2}\left(A r^{2}-2 B r+C\right)}{2 \Delta}\right)\left(-k_{4}+\frac{k_{4}^{2}(A r-B)}{\Delta}\right)=0
\end{aligned}
$$

or

$$
\exp \left(-\left(k_{3}-k_{4}\right) r+\frac{\left(k_{3}^{2}-k_{4}^{2}\right)\left(A r^{2}-2 B r+C\right)}{2 \Delta}\right)=-\frac{k_{4} \Delta-k_{4}^{2}(A r-B)}{k_{3} \Delta-k_{3}^{2}(A r-B)}
$$

Obviously, the left side of the above equation must be larger than zero, then $\frac{k_{4} \Delta-k_{4}^{2}(A r-B)}{k_{3} \Delta-k_{3}^{2}(A r-B)}<0$, i.e. $\left[k_{4} \Delta-k_{4}^{2}(A r-B)\right]\left[k_{3} \Delta-k_{3}^{2}(A r-B)\right]<0$. Arrange it and come to

$$
A^{2} r^{2}-\left(2 A B+\frac{\left(k_{3}+k_{4}\right) \Delta A}{k_{3} k_{4}}\right) r+\frac{\left(k_{3}+k_{4}\right) \Delta B+\Delta^{2}}{k_{3} k_{4}}+B^{2}<0 .
$$

Then we can discuss the solution of the quadratic inequality as follows:

(1) When $k_{3}>k_{4}$, then solution is $\frac{B}{A}+\frac{\Delta}{A k_{3}}<r<\frac{B}{A}+\frac{\Delta}{A k_{4}}$.

(2) When $k_{3}<k_{4}$, then solution is $\frac{B}{A}+\frac{\Delta}{A k_{4}}<r<\frac{B}{A}+\frac{\Delta}{A k_{3}}$.

Therefore, we can draw such a conclusion:

$$
\frac{B}{A}+\frac{\Delta}{A \max \left(k_{3}, k_{4}\right)}<r<\frac{B}{A}+\frac{\Delta}{A \min \left(k_{3}, k_{4}\right)} .
$$

Then we discuss the solution of equation (6) in the above mentioned interval.

(1) When $k_{3}>k_{4}$, equation (6) becomes

$$
\exp \left(-\left(k_{3}-k_{4}\right) r+\frac{\left(k_{3}^{2}-k_{4}^{2}\right)\left(A r^{2}-2 B r+C\right)}{2 \Delta}\right)=\frac{k_{4} \Delta-k_{4}^{2}(A r-B)}{-k_{3} \Delta+k_{3}^{2}(A r-B)} \text {. }
$$

Take the logarithms of both sides of the equation and get

$$
\begin{aligned}
\frac{k_{3}^{2}-k_{4}^{2}}{2 \Delta} A r^{2} & -\left(k_{3}-k_{4}+\frac{k_{3}^{2}-k_{4}^{2}}{\Delta} B\right) r+\frac{k_{3}^{2}-k_{4}^{2}}{2 \Delta} C \\
& =\ln \left(k_{4} \Delta-k_{4}^{2}(A r-B)\right)-\ln \left(k_{3}^{2}(A r-B)-k_{3} \Delta\right) .
\end{aligned}
$$

Firstly, we examine the solution of equation (3.6). If we let the left side be $y_{1}$ and the right side be $y_{2}$, then $y_{1}$ is a parabola with upward openings, whose symmetry axis is $r=\frac{B}{A}+\frac{\Delta}{A\left(k_{3}+k_{4}\right)}$. Based on the condition $r=\frac{B}{A}+\frac{\Delta}{A\left(k_{3}+k_{4}\right)}<$ $\frac{B}{A}+\frac{\Delta}{A k_{3}}$, we can know $y_{1}$ is increasing within the interval $\frac{B}{A}+\frac{\Delta}{A k_{3}}<r<$ $\frac{B}{A}+\frac{\Delta}{A k_{4}}$. And as $y_{2}^{\prime}=-\frac{k_{4}^{2} A}{k_{4} \Delta-k_{4}^{2}(A r-B)}-\frac{k_{3}^{2} A}{k_{3}^{2}(A r-B)-k_{3} \Delta}<0$, we can know $y_{2}$ is decreasing within the interval $\frac{B}{A}+\frac{\Delta}{A k_{3}}<r<\frac{B}{A}+\frac{\Delta}{A k_{4}}$. Consequently, $y_{1}$ and $y_{2}$ are bound to have one intersection point in the interval $\frac{B}{A}+\frac{\Delta}{A k_{3}}<r<\frac{B}{A}+\frac{\Delta}{A k_{4}}$, as shown in Figure 2. 


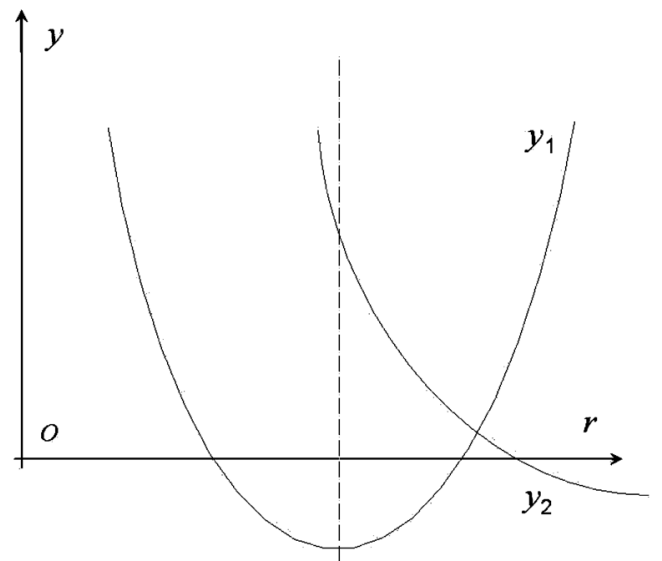

FiguRE 2. Determination of the root when $k_{3}>k_{4}$.

(2) When $k_{3}<k_{4}$, equation (3.4) is

$$
\exp \left(-\left(k_{3}-k_{4}\right) r+\frac{\left(k_{3}^{2}-k_{4}^{2}\right)\left(A r^{2}-2 B r+C\right)}{2 \Delta}\right)=\frac{k_{4}^{2}(A r-B)-k_{4} \Delta}{k_{3} \Delta-k_{3}^{2}(A r-B)} \text {. }
$$

Take the logarithms of both sides of the equation and get

$$
\begin{aligned}
\frac{k_{3}^{2}-k_{4}^{2}}{2 \Delta} A r^{2} & -\left(k_{3}-k_{4}+\frac{k_{3}^{2}-k_{4}^{2}}{\Delta} B\right) r+\frac{k_{3}^{2}-k_{4}^{2}}{2 \Delta} C \\
& =\ln \left[k_{4}^{2}(A r-B)-k_{4} \Delta\right]-\ln \left[k_{3} \Delta-k_{3}^{2}(A r-B)\right] .
\end{aligned}
$$

Firstly we examine the solution of equation (3.7). Let the left side be $y_{3}$ and the right side be $y_{4}$. Then $y_{3}$ is a parabola with downward openings, whose symmetry axis is $r=\frac{B}{A}+\frac{\Delta}{A\left(k_{3}+k_{4}\right)}$. As $r=\frac{B}{A}+\frac{\Delta}{A\left(k_{3}+k_{4}\right)}<\frac{B}{A}+\frac{\Delta}{A k_{4}}$, then we can know $y_{3}$ is monotonously decreasing in the interval $\frac{B}{A}+\frac{\Delta}{A k_{4}}<r<\frac{B}{A}+\frac{\Delta}{A k_{3}}$. And as $y_{4}^{\prime}=-\frac{k_{4}^{2} A}{k_{4}^{2}(A r-B)-k_{4} \Delta}+\frac{k_{3}^{2} A}{k_{3} \Delta-k_{3}^{2}(A r-B)}>0$, we can know $y_{4}$ is monotonously increasing in the interval $\frac{B}{A}+\frac{\Delta}{A k_{4}}<r<\frac{B}{A}+\frac{\Delta}{A k_{3}}$. So $y_{3}$ and $y_{4}$ are bound to have one intersection point in the interval $\frac{B}{A}+\frac{\Delta}{A k_{4}}<r<\frac{B}{A}+\frac{\Delta}{A k_{3}}$, as shown in Figure 3. Therefore, we can conclude that there must be one root of equation. Moreover, we can also prove that

$$
\begin{aligned}
\frac{\mathrm{d} z}{\mathrm{~d} r}= & \exp \left(-k_{3} r+\frac{k_{3}^{2}\left(A r^{2}-2 B r+C\right)}{2 \Delta}\right)\left(-k_{3}+\frac{k_{3}^{2}(A r-B)}{\Delta}\right)^{2} \\
& +\exp \left(-k_{3} r+\frac{k_{3}^{2}\left(A r^{2}-2 B r+C\right)}{2 \Delta}\right) \frac{k_{3}^{2} A}{\Delta} \\
& +\exp \left(-k 4 r+\frac{k_{4}^{2}\left(A r^{2}-2 B r+C\right)}{2 \Delta}\right)\left(-k_{4}+\frac{k_{4}^{2}(A r-B)}{\Delta}\right)^{2} \\
& +\exp \left(-k 4 r+\frac{k_{4}^{2}\left(A r^{2}-2 B r+C\right)}{2 \Delta}\right) \frac{k_{4}^{2} A}{\Delta}>0 .
\end{aligned}
$$




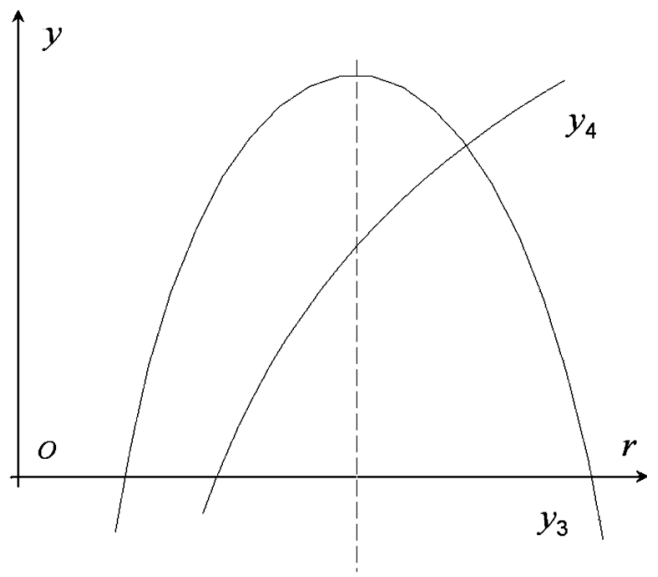

FiguRE 3. Determination of the root when $k_{3}<k_{4}$.

So $z$ is increasing and can come to zero, and as $k_{2}>0$ is one horizontal line, equation (5) is bound to have one root, i.e., we can get the expected return $r$ which maximizes $E U$. However, the equation is not a normal equation, so we fail to find its analytical solution. Thus we may try the numerical method for the solution. After gaining the expected return $r$, we can get its optimal investment proportion according to Formula (1) in the Mean-Variance Model.

\section{Actual application}

Suppose there are five kinds of securities. Their expectation return is $\mu=(0.208$

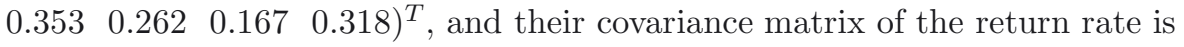

$$
\Sigma=\left(\begin{array}{ccccc}
2.10 & 2.21 & -2.16 & 1.62 & 2.15 \\
2.21 & 2.75 & -2.46 & 1.89 & -2.47 \\
-2.16 & -2.46 & 2.56 & -1.85 & 2.54 \\
1.62 & 1.89 & -1.85 & 1.42 & -1.88 \\
-2.15 & -2.47 & 2.54 & -1.88 & 2.66
\end{array}\right)
$$

From these data, we can get

$$
A=46.5979, \quad B=9.6042, \quad C=2.1580, \quad \Delta=8.3204 .
$$

\subsection{General CASe}

When $k_{2} \neq 0$ and $k_{3} \neq k_{4}$, without loss of generality, let $k_{1}=k_{2}=k_{3}=1$ and $k_{4}=2$, the utility function then becomes

$$
U=1+x-\mathrm{e}^{-x}-\mathrm{e}^{-2 x}
$$


and we can come to its expected utility function expressed as follows:

$$
\begin{aligned}
E U= & 1+r-\exp \left(-r+\frac{46.5979 r^{2}-19.2084 r+2.1580}{16.6408}\right) \\
& -\exp \left(-2 r+\frac{8685.4571 r^{2}-76.8336 r+8.3204}{16.6408}\right) .
\end{aligned}
$$

After this, we can get its maximum point $r=0.3670$ with non-constraint nonlinear programming model, and then get the optimal proportion based on Formula (2.1),

$$
\omega=(0.0415,0.6647,0.1026,-0.2390,0.4298)^{T} .
$$

\subsection{Three SPecial CASES}

Case 1. Double exponential utility function

Under the condition of $k_{2}=0$, the utility function becomes double exponential utility function [7]. Without loss of generality, let $k_{1}=1, k_{3}=1, k_{4}=2$, then the function becomes $U=1-\mathrm{e}^{-x}-\mathrm{e}^{-2 x}$, and the expected utility function is

$$
\begin{aligned}
E U= & 1+r-\exp \left(-r+\frac{46.5979 r^{2}-19.2084 r+2.1580}{16.6408}\right) \\
& -\exp \left(-2 r+\frac{8685.4571 r^{2}-76.8336 r+8.3204}{16.6408}\right),
\end{aligned}
$$

After this, we can get its maximum point $r=0.3141$ with non-constraint nonlinear programming model, and then get the optimal proportion based on Formula (2.1):

$$
\omega=(0.0462,0.3785,0.1563,0.0866,0.3320)^{T} \text {. }
$$

Case 2. Linear plus exponential utility function

Under the condition of $k_{2} \neq 0$, and either $k_{3}$ or $k_{4}$ is equal to 0 , then the utility function becomes the linear plus exponential utility function [8]. Without loss of generality, let $k_{1}=1, k_{2}=1, k_{3}=1, k_{4}=0$, then the function becomes $U=1+x-\mathrm{e}^{-x}$, and we can get its expected utility function is

$$
E U=1+r-\exp \left(-r+\frac{46.5979 r^{2}-19.2084 r+2.1580}{16.6408}\right) .
$$

After this, we can get its maximum point $r=0.5945$ with non-constraint nonlinear programming model, and obtain the optimal proportion based on Formula (2.1):

$$
\omega=(0.0241,1.7030,-0.0921,-1.4200,0.7847)^{T} .
$$

Case 3. Exponential utility function

Without loss of generality, under the condition of $k_{1}=1, k_{2}=0, k_{3}=1, k_{4}=1$, the utility function becomes the exponential utility function $U=1-2 \mathrm{e}^{-x}$ [9], and we can obtain its expected utility function is

$$
E U=1-2 \exp \left(-r+\frac{46.5979 r^{2}-19.2084 r+2.1580}{16.6408}\right) .
$$


After this, we can get its maximum point $r=0.3847$ with non-constraint nonlinear programming model, and then get the optimal proportion based on Formula (2.1):

$$
\omega=(0.0401,0.7455,-0.3308,-0.2390,0.4574)^{T} \text {. }
$$

\section{Conclusions And Final Remarks}

By employing NDCM, we have figured out the solution to the optimal decisionmaking of investors who apply the linear plus double exponential utility function. Moreover, we have obtained the optimal portfolio investment proportion. Meanwhile, there are still some problems for further exploration. For example, we take the hypothesis that the investment returns follow a Guass distribution, which is just a theoretical assumption. As a matter of fact, the investment returns just approximately follow a Guass distribution and even sometimes it may be very far from Guass distribution. Additionally, the parameters in the utility function call for more statistical data for fitting. We still need to make more efforts on these problems for solutions.

Acknowledgements. The authors are very indebated to Professor Huong Fuentes, Professor A.Ridha Mahjoub and also gratefully acknowledge the helpful comments and suggestions of the reviewers, which have improved our work.

\section{REFERENCES}

[1] X.H. He and Y. Chen, Utility Function and Economic Agent: An Insight from Financial Economics. The Theory and Practice of Finance and Economics 155 (2008) 1-7.

[2] H. Markowitiz, Portfolio selection. J. Finance 7 (1952) 77-91.

[3] Operational Research, Tsinghua University Press, Beijing (2005).

[4] W.F. Sharpe, G.J. Alexander and J.V. Bailey, Investments, 6th edn. Prentice-Hall International inc. New Jersey (1999).

[5] Z.X. Ye and J.Z. Lin, Mathematic FinanceAsset Pricing and Finance Decision-making Theory. Science Press, Beijing (1998).

[6] Y.T. Zhang, Statistic Analysis of Finance market, Guangxi Normal University Press, Guilin (1998).

[7] Q.J. Zhou, S.Y. Lv and J. Jiao, Decision-making of portfolio investment with double exponential utility function. J. Dalian University of Technology 51 (2011) 766-770.

[8] Q.J. Zhou and K.Y. Zhang, Research on Portfolio Investment with Linear plus Exponential Utility Function. Appl. Math. 17 (2004) 53-58.

[9] Q.J. Zhou and J.M. Wu, Two Methods of Optinal Portfolio Investment with Negative Exponential Utility Function and their Consistency. J. Dalian Nationalities University 6 (2004) 7-10. 